Z. Klin. Chem. Klin. Biochem.

13. Jg. 1975, S. 89-96

\title{
Über ein maschinelles enzymatisches Verfahren zur Bestimmung der Harnsäure
}

\author{
Von $P$. Hunziker
}

\section{F. Hoffman-La Roche \& Co. AG Diagnostica Schweizerhalle/Basel und}

\section{H. Keller}

Klin. chem. Zentrallabor Kantonsspital St. Gallen

(Eingegangen am 24. September 1974)

\begin{abstract}
Es wird ein Continuous Flow Verfahren zur Bestimmung von Harnsäure im Serum mit Uricase/Peroxidase und o-Dianısidin als Chromogen beschrieben. Die Methode kann sowohl auf sog. Einkanalsystemen, wie auch auf dem Mehrkanal-System SMA $12 / 60$ (Technicon) eingesetzt werden.

Das neue Verfahren wurde mit sechs verschiedenen anderen, teils manuellen, teils maschinellen Methoden zur Harnsäurebestimmung verglichen; die Resultate wurden statistisch ausgewertet. Es zeigte sich, daß alle enzymatischen Bestimmungsmethoden gut vergleichbare Resultate lieferten, während „chemische Oxidationsverfahren" systematische Abweichungen ergaben.
\end{abstract}

\section{A mechanized enzymic method for the determination of uric acid}

A continuous flow method is described for the determination of uric acid in serum using uricase and peroxidase, with $o$-dianisidine as the chromogen. The method can be used with single channel systems and with the multichannel system SMA 12/60 (Technicon).

The new method was compared with six other manual and mechanized methods for the dosage of uric acid, and the results were analyzed statistically. The results from all the enzymic methods showed a good correlation, whereas the "chemical oxidation" methods showed systematic deviations.

Das Prinzip der enzymatischen Harnsäure-UV-Bestimmung mit Uricase wurde 1941 von Bulger \& Johns (1) erstmalig beschrieben. 1947 wurde es von Kalckar (2) für das klinische Laboratorium adaptiert und seither in verschiedenen Modifikationen $(4,5,6)$ eingesetzt.

Für die Visualisierung der Uricase-Methode sind verschiedene Prinzipien bekannt. Das älteste ist das 1939 von Blauch \& Koch (7) vorgeschlagene indirekte Verfahren, das auf der Kombination mit der PhosphorwolframsäureMethode beruht. Für diese Bestimmung sind später mehrere Verbesserungen bekannt geworden $(8,9)$. Ein anderes Prinzip verläuft über den Nachweis des bei der Harnsäure-Oxidation entstehenden Wasserstoffperoxid, wobei $o$-Dianisidin in Gegenwart von Peroxidase in den bekannten rotbraunen Farbstoff überfihrt wird $(10,11)$. Diese Methode setzt eine vorherige Enteiweißung voraus. Beim erstmals von Kageyama vorgeschlagenen Verfahren (12) wird das Wasserstoffperoxid durch Methanol - unter Beteiligung von Katalase - reduziert, der entstehende Formaldehyd mit Acetylaceton und Ammonium-Ionen zum 3.5-Diacetyl-1.4-dihydrolutidin umgesetzt. Eine Enteiweißung ist nicht erforderlich (13).

Die automatisierte Harnsäure-Bestimmung wird bis heute fast ausschließlich mit Reduktionsmethoden durchgefuihrt. So wurde beim Du Port „ACA“ bis vor kurzem die Reduktion von $\mathrm{Cu}[\mathrm{II}]-2,2^{\prime}$-biscinchoninsäure-Chelat (14) herangezogen, infolge der unbefriedigenden Richtigkeit mittlerweile aber durch eine kinetische Uricase-UVMethode ersetzt (15).

Die Harnsäure-Bestimmung auf dem AutoAnalyzer (16, 17) wird heute noch im Prinzip mit Hilfe der 1912 von Folin und Denis (18) beschriebenen Phosphorwolframsäure-Reaktion durchgeführt. Da andere im Blut vorhandene reduzierend wirkende Verbindungen stören (19, 20), sind die so ermittel ten Harnsäure-Konzentrationen nicht vergleichbar mit Werten, die (auf manuellem Wege) mit Uricase-Verfahren gewonnen werden.

Das von Gochman \& Schmitz (21) beschriebene enzymatische Continuous Flow Verfahren zur Harnsäurebestimmung hat offenbar nicht die zu erwartende Verbreitung gefunden und nicht zur industriellen Fertigung einer entsprechenden Testpackung geführt. Ein von uns etwa gleichzeitig entwickeltes, ähnliches Continuous Flow System wurde mit sechs verschiedenen anderen heute üblichen Verfahren verglichen, um zu prüfen, ob es an Spezifität und Richtigkeit anderen Methoden nicht nachsteht. Durch ausgiebige Erfahrungen im Routinebetrieb sollte geklärt werden, ob seine Anwendung empfehlenswert ist. 


\section{Experimentelles}

Neu entwickeltes Continuous Flow Uricase/PeroxidaseVerfahren ${ }^{1}$ )

1. Puffer/Uricase-Lösung: $10 \mathrm{U}^{\text {Uricase }}{ }^{2}$ ) wurden in einem Liter Tetraborat-Puffer $(10 \mathrm{mmol} / 1, \mathrm{pH} 9,5)$ gelöst. Nach klarer Lösung wurden noch $10 \mathrm{ml}$ Tween 20 als Detergens zugefügt.

2. Puffer/Peroxidase-Lösung: In $250 \mathrm{ml}$ Phosphat-Puffer ( $240 \mathrm{mmol} / \mathrm{l}, \mathrm{pH} \mathrm{7,0)}$ wurden $2,5 \mathrm{mg}$ Peroxidase $\left.{ }^{3}\right)(55 \mathrm{U} / \mathrm{mg})$ gelöst und anschließend mit $2,5 \mathrm{ml}$ Tween 20 versetzt.

3. Chromogen-Lösung: $209,2 \mu \mathrm{mol} O$-Dianisidin-hydrochlorid wurden in $50 \mathrm{ml}$ bidest. Wasser gelöst und in dunkler Flasche aufbewahrt.

4. Verdünnungslösung: $2,5 \mathrm{ml}$ Tween 20 wurden in $1000 \mathrm{ml}$ $22,2 \mathrm{mmol} / 1 \mathrm{NaCl}$-Lösung gelöst.

5. Wäßrige Primär-Standards: Es wurde eine $5,95 \mathrm{mmol} / 1$ Harnsäurelösung hergestellt, deren aktueller Gehalt durch Kontrolle der Extinktion bei $\lambda=293 \mathrm{~nm}$ - nach vorheriger hundertfacher Verdünnung - festgelegt wurde. Der molare Absorptions-Koeffizient der Harnsäure beträgt $\epsilon_{293}=12,6$ $\cdot 10^{6} \mathrm{~cm}^{2} / \mathrm{mol}$.

Das Fließdiagramm ist in Abbildung 1 dargestellt; der Probentakt beträgt $60 / \mathrm{min}$.

Das Verfahren wurde auf AutoAnalyzer-Modellen der ersten und der zweiten Generation erprobt und schließlich endgültig in einen SMA $12 / 60$ eingebaut. Die Probennahme beträgt $60-1 / 1$ bis 60-9/1. Als Dialy sator wird ein 12- oder (besser) 24-inch-Modell verwendet, die Inkubationsdauer muß mindestens $4 \mathrm{~min}$ bei $37^{\circ} \mathrm{C}$ betragen. Der gebildete Farbstoff wird bei einer. Wellenlänge zwischen 420 und $460 \mathrm{~nm}$ gemessen.

\section{Vergleich s-Verfahren}

Carbonat-Phosphorwolframsäure-Methode nach Folin \& Denis (18) in der Modifikation von Caraway (22). Arbeitsvorschrift und alle Reagenzien wurden dem Harleco-Set Uric Acid (Best.Nr. 64148) entnommen.

AutoAnalyzer-Phosphorwolframsäure-Methode (HydroxylaminMethode) nach 1. c. (16). Die Reagenzien waren entsprechend dieser Vorschrift zubereitet, das Fließ-Schema entsprach der Hersteller-Vorschrift.

Uricase-Endpunktmessung nach Praetorius \& Poulsen (3). Als Photometer diente ein PMQ-2 (Zeiss Oberkochen), gemessen wurde bei $293 \mathrm{~nm}$. Alle Reagenzien wurden der BoehringerTestkombination Harnsäure (Bestell-Nr. 15986) entnommen.

Direkte kinetische Uricase-Methode, Du Pont-ACA-Verfahren (15). Die Arbeitsvorschrift und alle erforderlichen Reagenzien wurden en tsprechend der Hersteller-Vorschrift eingesetzt.

Kombinierte Phosphorwolframsäure/Uricase-Methode „CarawayMarable-Verfahren" (8), in der Modifikation von Eisenwiener \& Ferrat (9). Arbeitsvorschrift und alle Reagenzien wurden der Testpackung Harnsäure von Roche Diagnostica, Art.-Nr. 1063, entnommen.

Uricase/Katalase Verfahren nach Kageyama (12), die Arbeitsvorschrift und alle erforderlichen Reagenzien wurden der Boehringer Packung „Uricaquant" (Bestell-Nr. 15865) entnommen.

Testmaterialien und Analysengut

Wäßrige Primär-Standards: s. o.

Kommerzielle Kontroll-Seren: siehe Tabelle 2.

Patienten-Plasmen des Kantonsspitals St. Gallen: Blutentnahmen mittels Monovetten (23), die mit Ammoniumheparinat imprägniert waren. Alle Blutproben wurden innerhalb $1 \mathrm{~h}$

1) Testpackung Harnsäure (Uricase/POD) Continuous Flow von Roche Diagnostica, Art. Nr. 1502.

2) Uricase, Urate:oxygen oxidoreductase, EC 1.7.3.3.

3) Peroxidase, Donor:hydrogen-peroxide oxidoreductase, EC 1.11.1.7.

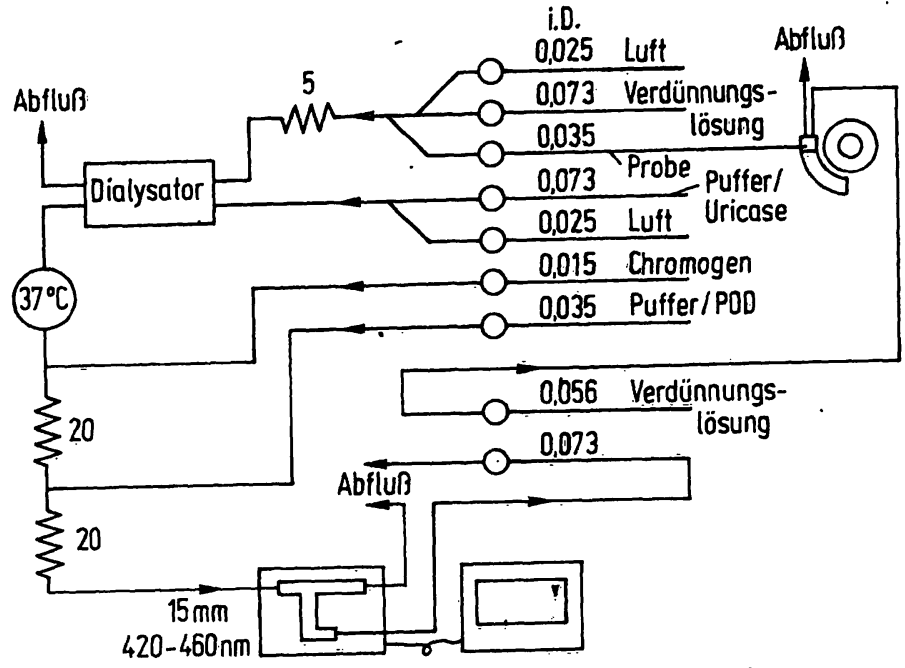

Abb. 1. Fließdiagramm zum Uricase/Peroxidase-Verfahren. Einzelheiten siehe Text.

nach der Blutentnahme zentrifugiert und das Plasma abgetrennt. Wenn nicht ausdrücklich anders erwähnt, wurde dieses Untersuchungsgut eingesetzt.

Urämie-Plasmen (Kantọnsspital St. Gallen): Entnahmen wie vorstehend. Als urämisch gelten Plasmen mit Harnstoff-Konzentrationen über $12,5 \mathrm{mmol} / 1$ ( $75 \mathrm{mg}$ Harnstoff $/ 100 \mathrm{ml})$ und Creatinin-Gehalten über $0,133 \mathrm{mmol} / 1$ (1,5 mg Creatinin/ $100 \mathrm{ml}$.

Lipämie-Plasmen (Kantonsspital St. Gallen): Blutentnahmen wie vorstehend. Als lipämisch galten solche Plasmen, die visuell milchig trüb waren und deren Triglyceridkonzentration eindeutig weit oberhalb des sog. Normalbereiches lagen (Tab. 8).

Probanden-Plasmen der „Studie Uzwil“ (25): Blutentnahmen wie bei den Spitalpatienten, die Blutproben wurden innerhalb von $2 \mathrm{~h}$ nach der Blutentnahme zentrifugiert und das Plasma abgetrennt.

„Studie Bad Ragaz“: Blutentnahme bei Patienten eines Ambulatoriums wie oben.

„Spital Liestal“ und „Basler Studie“: Konventionelle Blutentnahme mittels Record-Spritzen, Überführen des Blutes in Glas-Zentrifugen-Röhrchen, nach abgelaufener Gerinnung Zentrifugieren mit anschließender Separierung des Serums.

\section{Ergebnisse}

\section{Linearitätsbereich}

Der Linearitätsbereich des neuentwickelten Verfahrens wurde mit wäßrigen Verdünnungsreihen von Harnsäure-Lösungen geprüft. Es zeigte sich, daß bis $2400 \mu \mathrm{mol} / 1$ lineare Absorptionsdifferenzen ermittelt werden konnten. Die Tabelle 1 gibt das Ergebnis dieser Meß-Serie wieder.

Tab. 1. Linearität der Methode: Wäßrige Harnsäure-Standards der Konzentrationen 297, 595, 892, 1190, 1785 und $2380 \mu \mathrm{mol} / \mathrm{l}$ wurden wie Proben eingesetżt und die Ergebnisse auf den Standard $892 \mu \mathrm{mol} / 1$ (S) bezogen. Die Meßreihe wurde mit einem 24 inch-Diạlysator und einer Inkubationsdauer von $\mathbf{4} \mathrm{min}$ durchgeführt.

\begin{tabular}{|c|c|c|c|c|c|c|}
\hline $\begin{array}{l}\text { Standard } \\
(\mu \mathrm{mol} / 1)\end{array}$ & 297 & 595 & 892 & 1190 & 1785 & 2380 \\
\hline $\begin{array}{l}\text { Ergebnis } \\
(\mu \mathrm{mol} / 1)\end{array}$ & 282 & 571 & $\mathbf{S}$ & 1200 & 1810 & 2420 \\
\hline
\end{tabular}


Tab. 2. Vergleich der mit der Uricase/Peroxidase-Methode gefundenen Resultate mit den Deklarationswerten verschiedener Kontrollseren (Richtigkeit).

\begin{tabular}{|c|c|c|c|}
\hline Serum & $\begin{array}{l}\text { Deklaration } \\
{[\mu \mathrm{mol} / 1]}\end{array}$ & Methode & $\begin{array}{l}\text { Ergebnis } \\
{[\mu \mathrm{mol} / 1]}\end{array}$ \\
\hline $\begin{array}{l}\text { Hyland I } \\
0369 \text { R007 A1 }\end{array}$ & $\begin{array}{l}303(286-321) \\
303 \\
274\end{array}$ & $\begin{array}{l}\text { Phosphorwolframsäure, Beckman Uricase } \\
\text { AutoAnalyzer } \\
\text { Hycel Mark X }\end{array}$ & 274 \\
\hline $\begin{array}{l}\text { Hyland II } \\
0368 \text { T004 B1 }\end{array}$ & $\begin{array}{l}595(565-625) \\
595 \\
572 \\
595(559-636)\end{array}$ & $\begin{array}{l}\text { Phosphorwolframsäure, Beckman Uricase } \\
\text { AutoAnalyzer } \\
\text { Hycel Mark X } \\
\text { Harleco }\end{array}$ & 571 \\
\hline $\begin{array}{l}\text { Seronorm } \\
118\end{array}$ & $\begin{array}{l}345 \\
399 \\
321 \\
315 \\
321\end{array}$ & $\begin{array}{l}\text { AutoAnalyzer Lab. A } \\
\text { Uricase Lab. B } \\
\text { Uricase Lab. C } \\
\text { Uricase Lab. D } \\
\text { Uricase Lab. E }\end{array}$ & 333 \\
\hline $\begin{array}{l}\text { Monitrol I } \\
114 \mathrm{~B}\end{array}$ & $\begin{array}{l}286 \\
321 \\
297 \\
315 \\
315\end{array}$ & $\begin{array}{l}\text { Folin } \\
\text { Kern-Stransky, Lab. A } \\
\text { Kern-Stransky, Lab. B } \\
\text { Caraway } \\
\text { AutoAnalyzer }\end{array}$ & 291 \\
\hline $\begin{array}{l}\text { Monitrol II } \\
27 \mathrm{~A}\end{array}$ & $\begin{array}{l}482 \\
518 \\
494 \\
506 \\
500\end{array}$ & $\begin{array}{l}\text { Folin } \\
\text { Kern-Stransky, Lab. A } \\
\text { Kern-Stransky, Lab. B } \\
\text { Caraway } \\
\text { AutoAnalyzer }\end{array}$ & 488 \\
\hline
\end{tabular}

Bei Lagerung der Uricase-Lösung bei Raumtemperatur nimmt der Linearitätsbereich ab, nach 28 Tagen ist er auf etwa $1200 \mu \mathrm{mol} / 1$ abgesunken. Wird die PufferUricase-Lösung dagegen bei $+4{ }^{\circ} \mathrm{C}$ im Kühlschrank gelagert, so ist auch nach 4 Wochen noch keine Veränderung des Linearitätsbereiches meßbar.

\section{Richtigkeit}

Die Richtigkeit wurde mit Hilfe von käuflichen Kontrollseren überprüft. Dabei wurde das Ergebnis an Hand eines wäßrigen Primärstandards, dessen Konzentration $297 \mu \mathrm{mol} / 1$ betrug, berechnet. Das Ergebnis zeigt die Tabelle 2, die mit der neuen Methode ermittelten Resultate stimmen mit den deklarierten Resultaten gut überein.

\section{Präzision}

Die Präzision ,in der Serie“ wurde an 5 verschiedenen kommerziellen Kontrollseren geprüft. Standardabweichung und Variations-Koeffizient gehen aus der Tabelle 3 hervor.

Die Präzision ,von Tag zu Tag", beobachtet über die ersten 7 Monate des Jahres 1974, wobei an sechs Tagen der Woche Präzisionskontrollen durchgeführt wurden, zeigt die Tabelle 4.
Tab. 3. Präzision ,,in der Serie“. Reproduzierbarkeit der Methode bei verschiedenen Kontrollseren.

$\overline{\mathbf{x}}=$ Mittelwert Harnsäure $[\mu \mathrm{mol} / 1]$

$s \quad=$ Standardabweichung $[\mu \mathrm{mol} / 1]$

VK = Variationskoeffizient (\%)

$\mathbf{N}=$ Anzahl der in der Serie durchgeführten Bestimmungen.

\begin{tabular}{lllll}
\hline Serum & $\bar{x}$ & $s$ & VK & N \\
\hline $\begin{array}{l}\text { Hyland I } \\
\text { 0369 R 007A1 }\end{array}$ & 268 & 3,2 & 1,20 & 15 \\
$\begin{array}{l}\text { Roche } \\
\text { C 1237 }\end{array}$ & 248 & 2,9 & 1,18 & 15 \\
$\begin{array}{l}\text { Hyland II } \\
\text { 0368 T002A1 }\end{array}$ & 542 & 0,7 & 0,13 & 10 \\
$\begin{array}{l}\text { Monitrol I } \\
\text { 119 AB } \\
\begin{array}{l}\text { Seronorm } \\
\text { 120 }\end{array}\end{array}$ & 261 & 1,9 & 0,73 & 10 \\
\hline
\end{tabular}

\section{Wiederfindung (,Recovery")}

Zwei kommerziellen Kontrollseren wurden gleiche Mengen wäßriger Standardlösung bekannter Konzentration zugefügt und die Wiederfindung berechnet. Die Ergebnisse sind in der Tabelle 5 zusammengestellt. 
Tab. 4. Präzision von „Tag zu Tag“, Ergebnisse der RoutineQualitätskontrolle in den ersten sieben Monaten des Jahres 1974.

$\overline{\mathbf{x}}=$ Mittelwert Harnsäure $[\mu \mathrm{mol} / 1]$

$s \quad=$ Standardabweichung $[\mu \mathrm{mol} / 1]$

VK $=$ Variationskoeffizient (\%).

\begin{tabular}{llll}
\hline Monat & $\overline{\mathbf{x}}$ & s & VK \\
\hline Januar & 260,2 & 8,3 & 3,18 \\
Februar & 263,7 & 9,9 & 3,77 \\
März & 293,9 & 5,4 & 1,84 \\
April & 296,5 & 4,8 & 1,63 \\
Mai & 292,3 & 8,0 & 2,75 \\
Juni & 290,1 & 7,3 & $2 ; 52$ \\
Juli & 287,1 & 10,9 & 3,79 \\
\hline
\end{tabular}

Tab. 5. Recovery-Untersuchungen.

$\mathrm{S}_{1}$ : Hyland I $0369 \mathrm{R} 007 \mathrm{~A} 1$

$S_{2}$ : Hyland II 0368 R001 A1

$\mathrm{H}_{1}$ : Harnsäure-Standard $55,3 \mu \mathrm{mol} / 1$

$\mathrm{H}_{2}$ : Harnsäure-S tandard $110,6 \mu \mathrm{mol} / 1$

$\mathrm{H}_{3}$ : Harnsäure-Standard $166 \mu \mathrm{mol} / 1$

Die lyophilisierten Kontrollseren wurden in Wasser, bzw.

in den Harnsäurelösungen $\mathrm{H}_{1}, \mathrm{H}_{2}$ und $\mathrm{H}_{3}$ aufgelöst.

\begin{tabular}{|c|c|c|c|c|}
\hline Lösung & & gefunden & Sollwert & Recovery \\
\hline $\begin{array}{l}S_{1} \\
S_{1}+H_{1} \\
S_{1}+H_{2} \\
S_{1}+H_{3} \\
S_{2} \\
S_{2}+H_{1} \\
S_{2}+H_{2} \\
S_{2}+H_{3}\end{array}$ & $\therefore:$ & $\begin{array}{l}268 \\
321 \\
377,5 \\
434 \\
533 \\
600 \\
654 \\
695\end{array}$ & $\begin{array}{l}323 \\
378 \\
433 \\
\\
588 \\
644 \\
699\end{array}$ & $\begin{array}{c}99,4 \\
99,9 \\
100,2 \\
\\
102 \\
101,5 \\
99,4\end{array}$ \\
\hline
\end{tabular}

\section{Verschleppung („Carry Over“)}

Für Continuous Flow Systeme läßt sich bekanntlich ein gewisser Verschleppungsfehler grundsätzlich nicht restlos vermeiden. Die quantitative Untersuchung dieses Fehlers nach der Methode von Hjelm (24) zeigt die Tabelle 6. Der erhaltene Interaktionskoeffizient $q=0,012$ beweist den geringen Störeinfluß durch Probenwechselwirkung.

\section{Interferenzen}

Auf eventuelle Störeinflüsse durch Verbindungen, die im strömenden Blut enthalten sind, wurde geprüft durch Auflösen von lyophilisierten Kontrollseren in wäßrigen Lösungen dieser Komponenten. Untersucht wurden derartige Lösungen folgender Verbindungen:

Allantoin

$630 \mu \mathrm{mol} / 1$

p-Aminobenzoesäureäthylester $600 \mu \mathrm{mol} / 1$

Ascorbinsäure $284 \mu \mathrm{mol} / 1$

Gentisinsäure

$47 \mu \mathrm{mol} / 1$

Glutathion

$325 \mu \mathrm{mol} / 1$

Creatinin

$440 \mu \mathrm{mol} / 1$

1-Phenyl-2,2-dimethyl-4-aminopyrazolon-(5) $98 \mu \mathrm{mol} / 1$

Die in der Tabelle 7 wiedergegebenen Ergebnisse zeigen, daß die Harnsäurebestimmung durch physiologische

Tab. 6. Quantitative Bestimmung des Interaktionskoeffizienten $q$ nach Hjelm (24)

$q=\frac{S_{4}-S_{2}}{S_{3}-S_{4}}=0,012$

$\mathrm{S}_{1}, \mathrm{~S}_{2}, \mathrm{~S}_{4}=$ Standard $178 \mu \mathrm{mol} / 1$ $S_{3}=$ Standard $952 \mu \mathrm{mol} / 1$.

\begin{tabular}{lllll}
\hline Probe & $S_{1}$ & $S_{2}$ & $S_{3}$ & $S_{4}$ \\
\hline $\begin{array}{l}\text { Harnsäure, } \\
\text { gefunden }[\mu \mathrm{mol} / \mathrm{l}]\end{array}$ & 174 & 178 & 928 & 187 \\
\hline
\end{tabular}

Tab. 7. Untersuchung auf mögliche Störeinflüsse.

\begin{tabular}{|c|c|c|c|c|}
\hline Serum & Zugefügte Fremdsubstanz & & $\begin{array}{l}\text { Sollwert } \\
\text { (umol/1) }\end{array}$ & $\begin{array}{l}\text { Gefunden } \\
(\mu \mathrm{mol} / \mathrm{l})\end{array}$ \\
\hline \multirow[t]{2}{*}{$\begin{array}{l}\text { Monitrol I } \\
119 \mathrm{AB}\end{array}$} & - & & 274 & 274 \\
\hline & $\begin{array}{l}\text { Allantoin } \\
\text { Glutathion } \\
\text { Creatinin }\end{array}$ & $\begin{array}{l}630 \mu \mathrm{mol} / 1 \\
325 \mu \mathrm{mol} / 1 \\
440 \mu \mathrm{mol} / 1\end{array}$ & $\begin{array}{l}274 \\
274 \\
274\end{array}$ & $\begin{array}{l}280 \\
274 \\
280\end{array}$ \\
\hline \multirow[t]{2}{*}{$\begin{array}{l}\text { Seronorm } \\
120\end{array}$} & - & & 303 & 303 \\
\hline & Ascorbinsäure & $284 \mu \mathrm{mol} / 1$ & 303 & 319 \\
\hline \multirow[t]{3}{*}{$\begin{array}{l}\text { Monitrol II } \\
33 \mathrm{AB}\end{array}$} & - & & $\begin{array}{l}577 \\
559 \\
654 \\
631 \\
601\end{array}$ & 523 \\
\hline & Gentisinsäure & $47 \mu \mathrm{mol} / 1$ & & 493 \\
\hline & $\begin{array}{l}\text { p-Aminobenzoesäureäthylester } \\
\text { 1-Phenyl-2,3-dimethyl-4-äminopyrazolon-(5) }\end{array}$ & $\begin{array}{r}600 \mu \mathrm{mol} / 1 \\
98 \mu \mathrm{mol} / \mathrm{l}\end{array}$ & & $\begin{array}{l}522 \\
495\end{array}$ \\
\hline
\end{tabular}


Tab. 8. Harnsäure-Bestimmung in stark lipämischen, milchig trüben Plasmen mit drei verschiedenen Methoden. Einzelheiten siche Text.

\begin{tabular}{cccccc}
\hline Nr. & Cholesterin & Triglyceride & \multicolumn{3}{l}{ Harnsäure $[\mu \mathrm{mol} / 1]$} \\
& {$[\mathrm{mmol} / \mathrm{l}$} & {$[\mathrm{mmol} / \mathrm{l}]$} & Roche & ACA & Kageyama \\
\hline 1 & 5,66 & 17,1 & 500 & 488 & 52 \\
2 & 6,98 & 13,8 & 286 & 303 & 17 \\
3 & 6,08 & 18,9 & 559 & 506 & negativ \\
4 & 6,72 & 8,8 & 321 & 291 & 17 \\
5 & 12,5 & 41,2 & 226 & 256 & 11 \\
6 & 21,8 & 30,4 & 512 & 434 & 36 \\
7 & 6,75 & 21,7 & 345 & 291 & 5,9 \\
8 & 13,8 & 44,4 & 589 & 529 & 23 \\
9 & 14,8 & 17,7 & 506 & 440 & negativ \\
10 & 9,83 & 20,6 & 535 & 506 & 36 \\
\hline
\end{tabular}

Konzentrationen ${ }^{4}$ ) dieser Fremdstoffe nicht wesentlich beeinflußt wird.

\section{Vergleichbarkeit mit anderen Verfahren zur Harnsäure-Bestimm ung}

Das beschriebene Uricase/Peroxidase-Verfahren wurde mit der ,klassischen“ Phosphorwolframsäure-Methode (Abb. 2), mit der manuellen (Abb. 3) und einer automatisierten (kinetischen) direkten Uricase UV-Methode und schließlich mit zwei anderen ,indirekten“ UricaseMethoden verglichen. Die erhaltenen Resultate sind in Tabelle 9 zusammengestellt.

4) Roseman \& Dorfman (30) geben als maximale Serum-Gentisinsäure Konzentration - nach oraler Verabreichung von $1,8 \mathrm{~g}$ Natriumsalicylat (entsprechend 6 Aspirintabletten) $18,7 \mu \mathrm{mol} / \mathrm{l}$ an.

Nach Brubacher (Basel, persönl. Mitt.) gilt für Probanden mit regelmäßiger Vitamin C-Einnahme folgender Serumascorbinsäure-Normalbereich: $\bar{x}=72,1 \mu \mathrm{mol} / 1, \mathrm{~s}=34,1 \mu \mathrm{mol} / 1$.

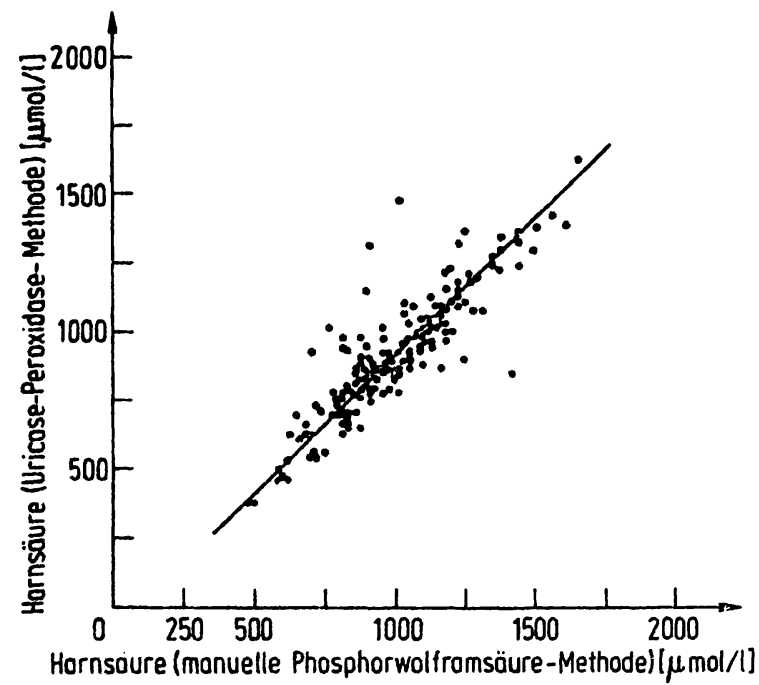

Abb. 2. Vergleich von Harnsäurebestimmungs-Methoden: Uricase/Peroxidase-Methode gegen manuelle Phosphorwolframsäure-Methode. Einzelheiten siche Text.

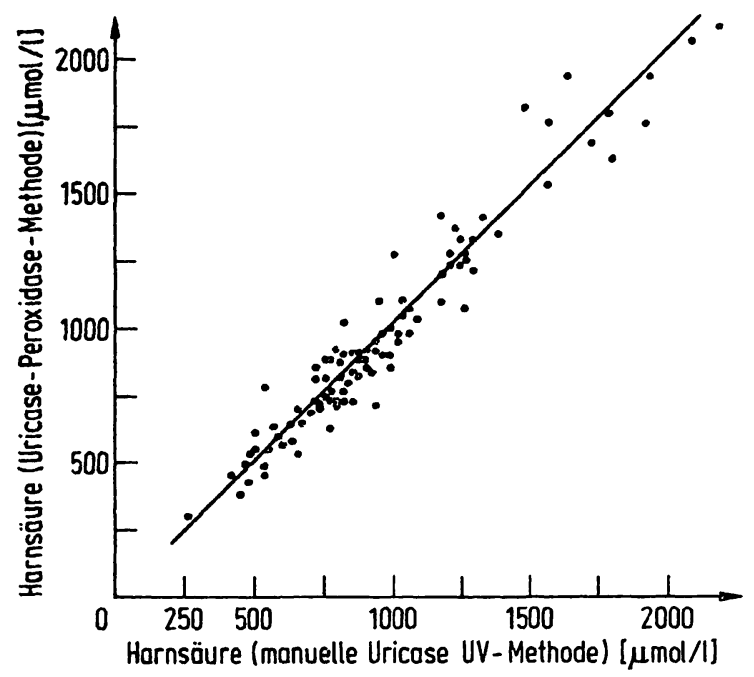

Abb. 3. Vergleich von Harnsäurebestimmungs-Methoden: Uricase/Peroxidase-Methode gegen manuelle Uricase UV-Methode.

Einzelheiten siehe Text.

Tab. 9. Übersicht Methodenvergleich.

\begin{tabular}{|c|c|c|c|c|c|}
\hline Methoden-Vergleich & $\mathbf{N}$ & $y=f(x)$ & $\mathbf{r}$ & $\begin{array}{l}\text { Sy } \\
(\mu \mathrm{mol} / \mathrm{l})\end{array}$ & $\begin{array}{l}\mathrm{Sx} \\
(\mu \mathrm{mol} / \mathrm{l})\end{array}$ \\
\hline $\begin{array}{l}\text { 1. Uricase-Peroxidase-/manuelle } \\
\text { Phosphorwolframsäure-Methode }\end{array}$ & $\begin{array}{l}199 \\
\text { (Basler Studie) }\end{array}$ & $\begin{aligned} & y= 1,006 x- \\
& 34,1 \mu \mathrm{mol} / 1\end{aligned}$ & 0,903 & 77,1 & 76,7 \\
\hline $\begin{array}{l}\text { 2. Uricase-Peroxidase-I } \\
\text { manuelle Uricase-UV-Methode }\end{array}$ & $\begin{array}{l}101 \\
\text { (Spital Liestal) }\end{array}$ & $\begin{aligned} y= & 1,041 x- \\
& 14,1 \mu \mathrm{mol} / 1\end{aligned}$ & 0,976 & 148,1 & 142,4 \\
\hline $\begin{array}{l}\text { 3. Uricase-Peroxidase-/direkte, } \\
\text { kinetische Uricase-UV-Methode }\end{array}$ & 20 & $\begin{aligned} & y= 1,002 x- \\
& 0,9 \mu \mathrm{mol} / 1\end{aligned}$ & 0,998 & 91,2 & 91,0 \\
\hline $\begin{array}{l}\text { 4. Uricase-Peroxidase-/Phosphor- } \\
\text { wolframsäure-Uricase-Methode }\end{array}$ & $\begin{array}{l}237 \\
\text { (Spital Liestal) }\end{array}$ & $\begin{aligned} & y= 0,926 x+ \\
& 12,2 \mu \mathrm{mol} / 1\end{aligned}$ & 0,948 & 135,9 & 146,1 \\
\hline $\begin{array}{l}\text { 5. Uricase-Peroxidase-I } \\
\text { Uricase-Katalase-Methode (Kageyama) }\end{array}$ & $\begin{array}{l}50 \\
\text { (Bad Ragaz-Studie) }\end{array}$ & $\begin{aligned} & y= 1,026 x- \\
& 8,0 \mu \mathrm{mol} / 1\end{aligned}$ & 0,987 & 94,7 & 92,3 \\
\hline
\end{tabular}


Ergebnisse und 'Vergleichbarkeit bei Blutproben von urämischen und hyperlipidämischen

\section{Patienten}

\section{Urämie-Plasmen}

Uricase/Peroxidase/o-Dianisidin-Verfahren, verglichen mit der AutoAnalyzer-Phosphorwolframsäure-Methode.

Für diese Vergleichsuntersuchungen standen die Plasmen von 105 verschiedenen Patienten zur Verfügung.

Die Resultate sind in der Abbildung 4 wiedergegeben. Man ersieht daraus, daß eine systematische Abweichung zwischen den Methoden besteht, wobei erwartungsgemäß die Resultate des Uricase/Peroxidase/o-DianisidinVerfahrens $120 \mu \mathrm{mol} / 1$ niedriger liegen. Die Ausgleichsgerade wurde in der Abbildung nicht eingetragen, da sie offensichtlich nicht befriedigt. Man hat den Eindruck, daß die höheren Harnsäure-Werte einer zweiten Ausgleichsgeraden zuzuordnen wären, bzw., daß etwa bei $x=450$ respektive $y=300$ ein Knick liegt. Tatsächlich werden auch sehr differente Ergebnisse erhalten, wenn man die höheren Harnsäure-Werte von den niedrigeren abtrennt und die Vergleichbarkeit der Methoden für diese beiden Kollektive getrennt untersucht. Man kann hier die Hypothese formulieren, daß die Beeinflussung der Phosphorwolframsäure-Methode durch unspezifische Chromogene je nach Schwere der Azotämie unterschiedlich sei. Diese Frage muß aber einer eigenen Untersuchung vorbehalten bleiben.

\section{Hyperlipidämie-Plasmen}

Uricase/Peroxidase/o-Dianisidin-Verfahren, verglichen mit dem Kageyama-Verfahren und dem ACA-Verfahren.

Von auswärtigen Laboratorien war uns berichtet worden, daß die Harnsäure-Bestimmung mit dem Kageyama-Verfahren auf Schwierigkeiten stößt, wenn es sich um lip-

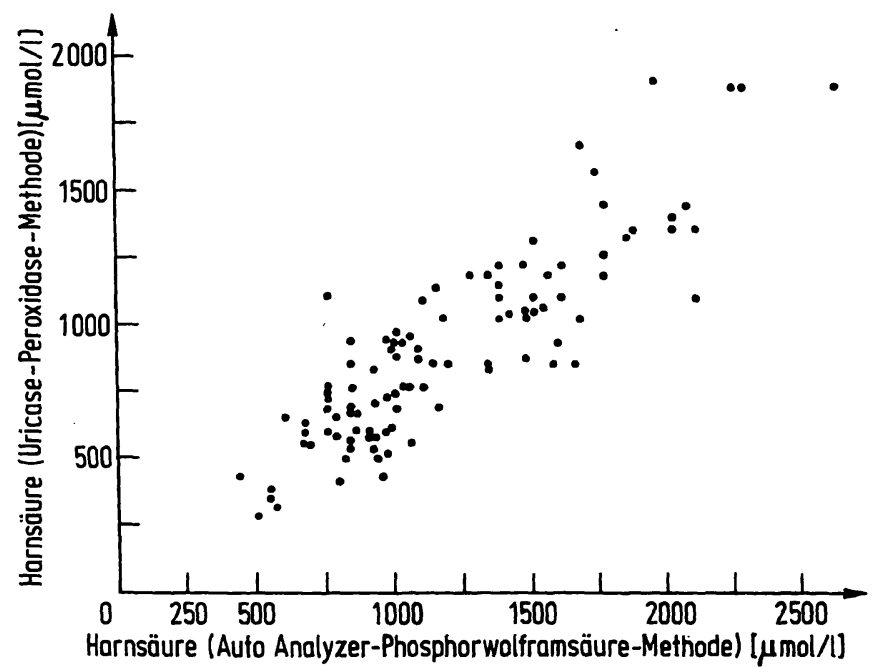

Abb. 4. Vergleich von Harnsäurebestimmungs-Methoden an urämischen Plasmen:

Uricase/Peroxidase-Methode gegen Autoanalyzer-Phosphorwolframsäure-Methode.

Einzelheiten siehe Text. ämische Plasmen handelt. Wir haben deshalb milchig trübe, lipämische Plasmen auf ihren Harnsäure-Gehalt untersucht und dabei das Kageyama-Verfahren, das ACA-Verfahren und das Uricase/Peroxidase/o-DianisidinVerfahren miteinander verglichen. Es standen uns insgesamt 10 Proben mit extremen Triglycerid- und teilweise erhöhten Cholesterin-Konzentrationen zur Verfügung. Die Resultate sind in der Tabelle 8 wiedergegeben. Man ersieht daraus, daß bei lipämischen Senen die Korrelation zwischen Uricase/Peroxidase/o-Dianisidin- und ACAVerfahren deutlich schlechter ist, als bei den klaren Seren, doch können die Werte als vergleichbar bezeichnet werden. Im Gegensatz dazu liefert die Kageyama-Methode unsinnig niedrige, in zwei Fällen sogar „negative“ Resultate.

Ermittlung des sog. Normalbereiches für PlasmaHarnsäure bei Männern in einem Feldversuch „Studie Uzwil“"

Von der Âbteilung für Präventiv-Medizin des Kantons St. Gallen ist 1973 eine Reihenuntersuchung in einem Schweizerischen Großbetrieb an insgesamt 1413 männlichen Arbeitern und Angestellten durchgeführt worden. Neben zahlreichen anderen klinischen und klinisch-chemischen Parametern wurde dabei mit dem Uricase/ Peroxidase/o-Dianisidin-Verfahren der Harnsäurespiegel im Nüchternplasma bestimmt. Das Histogramm und die Percentile sind in der Abbildung 5 wiedergegeben. Das arithmetische Mittel und die $2 \mathrm{~s}$-Grenzen betragen $279 \pm 145 \mu \mathrm{mol} / 1$ (134-424 $\mu \mathrm{mol} / \mathrm{l})$.

\section{Diskussion}

Das beschriebene Verfahren liefert vergleichbare Ergebnisse mit allen vier geprüften enzymatischen Methoden. Ob dabei die Extinktionsabnahme bei 293 nm direkt beobachtet wird oder ob die Oxidation durch Sekundärreaktionen visualisiert wird, ist für das Ergebnis offenbar ohne Bedeutung.

Im Gegensatz dazu bestehen systematische Unterschiede zu den Resultaten von nichtenzymatischen Phosphorwolframsäure-Verfahren (Tab. 9). Diese Unterschiede werden noch gravierender, wenn es sich um Seren urämischer Patienten handelt, da bei diesen derart falsch hohe Resultate gefunden werden, daß falsche klinische Interpretationen und Maßnahmen die Folge sein können.

Unsere Untersuchungen haben gezeigt, daß bei Verwendung käuflicher Kontrollseren die mangelhafte Spezifität der Oxidationsverfahren nicht unbedingt erkennbar ist, da das wechselnde. Verhältnis Harnsäure/unspezifische Chromogene der Patientenplasmen nicht ausreichend imitiert werden kann. Hierauf sind vermutlich die divergenten Äußerungen zurückzuführen, die auf dem IV. Internationalen Symposium über Qualitätskontrolle zur 


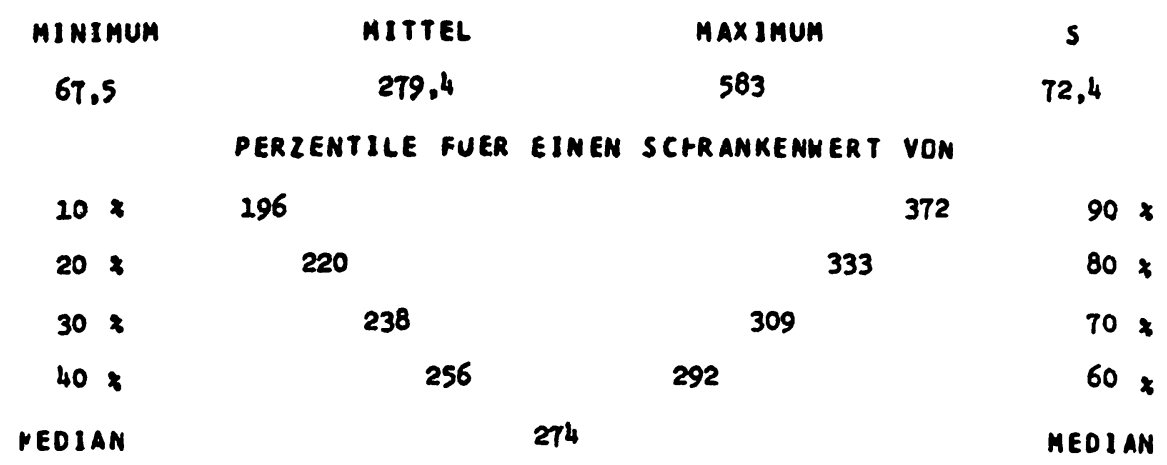

HARNSAEURE VON VS2 WENN MICHT VON VSB

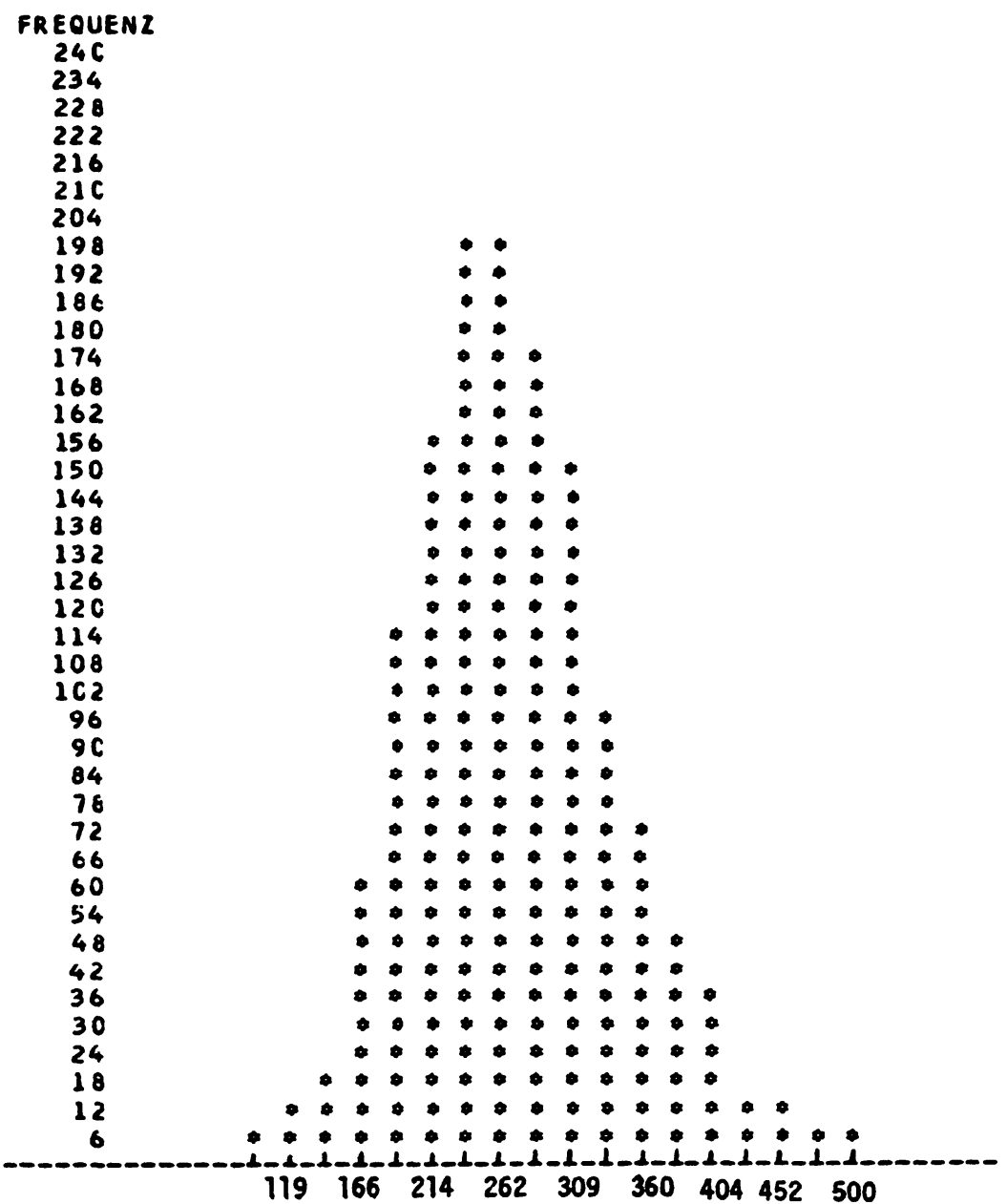

Abb. 5. Histogramm und Perzentile von Harnsäurebestimmungen mittels Uricase/Peroxidase-Methode an 1413 männlichen Probanden.

Einzelheiten siehe Text.

Härnsäurebestimmung gemacht worden sind (26). Es muß eindeutig festgestellt werden:

1. Wäßrige Harnsäurelösungen in Form von sog. Primärstandards, ergeben mit ,chemischen" Oxidationsmethoden wie mit Uricase-Methoden übereinstimmende Ergebnisse.

2. Humanplasmen/Seren enthalten stets unspezifische Chromogene, die mit den ,chemischen" Oxidationsverfahren eine Verfälschung des Wertes nach oben bewirken.
3. Der Anteil der unspezifischen Chromogene ist beim „Gesunden" offenbar relativ konstant und im Vergleich zur Harnsäure niedrig. Bei bestimmten Erkrankungen, insbesondere bei Nieren-Insuffizienzen, steigt jedoch der Anteil der unspezifischen Chromogene in einer nicht geringen $Z$ ahl von Fällen so stark an, daß grobe Fehlbestimmungen mit den unspezifischen Methoden resultieren.

4. Bei den direkten Uricase-Verfahren werden an die instrumentellen Bedingungen relativ hohe Anforderungen gestellt, wenn eine befriedigende Präzision erreicht werden soll. Sind die meßtechnischen Voraus- 
setzungen aber erfüllt, so arbeiten diese Verfahren störungsfrei und führen zu richtigen und präzisen Werten.

5. Die Visualisierung der Uricase-Reaktion mittels einer Katalase/- oder Peroxidase/Chromogen-Reaktion, kann durch verschiedene Faktoren gestört werden, z. B. ist die Kageyama-Methode gegen stärkere lipämische Trübungen empfindlich. Daß Peroxidase/Chromogen-Reaktionen durch andere Redox-Substanzen, insbesondere durch Ascorbinsäure gestört werden können, ist seit langem bekannt. Durch geeignete Bedingungen ist es aber möglich, diese Störungen auszuschalten oder so gering zu halten, daß sie für das Resultat irrelevant sind.

6. Die Wichtigkeit einer nicht nur präzisen, sondern auch richtigen Harnsäurebestimmung ist durch den Zusammenhang zwischen Harnsäure und Gicht gegeben.

Die Störung der Kageyama-Methode durch lipämische Trübungen wird weder in der Originalarbeit (12) noch in der deutschen Einführungsarbeit von Forstmayer (13) oder der vergleichenden Untersuchung von Mertz (27) erwähnt. Andererseits ist die Adaptation dieses Verfahrens auf das Continuous Flow System so lange nicht möglich, bis die Gesamtinkubationsdauer nicht auf $9 \mathrm{~min}$ - die in einem Mehrkanalsystem maximal zur Verfügung stehende Zeit - beschränkt werden kann.

Das beschriebene Verfahren hat sich in der Praxis ohne Einschränkung bewährt: Seit Januar 1973 sind damit über 50000 Harnsäurebestimmungen durchgeführt worden, ohne daß eine Beanstandung erfolgt ist.

\section{Literatur}

1. Bulger, H. A. \& Johns, H. E. (1941), J. Biol. Chem. 140, $427-440$.

2. Kalckar, H. M. (1947), J. Biol. Chem. 167, 429-443.

3. Praetorius, E. \& Poulsen, H. (1953), Scand. J. Clin. Lab. Invest. 5, 273-280.

4. Zöllner, N. (1963), diese Z. 1, 178-182.

5. Kortüm, M. \& Klin, O. (1972), Ärztl. Lab. 18, 33-36.

6. Da Fonseca-Wollheim, F. (1972), diese Z. 10, 136.

7. Blauch, M. B. \& Koch, F. C. (1939), J. Biol. Chem. 130, 443-454.

8. Caraway, W. T. \& Marable, H. (1966), Clin. Chem. 12, $18-24$.

9. Eisenwiener, H. G. \& Ferrat, R. (1974), Chem. Rundsch. 27, - No. 7, 19-21.

10. Lorentz, K. \& Berndt, W. (1967), Anal. Biochem. 18, 58-63.

11. Domagk, G. F. \& Schlicke, H. H. (1968), Anal. Biochem. 22, 219-224.

12. Kageyama, N. (1971), Clin. Chim. Acta 31, 421-426.

13. Forstmeyer, H. (1974), Ärtzl. Lab. 20, 125-132.

14. Gindler, E. M. (1970), Clin. Chem. 16, 536.

15. Lum, G. \& Gambino, S. R. (1973), Clin. Chem. 19, 1184 bis 1186 .

16. Musser, A. W. \& Ortigoza, C. (1966), Tech. Bull. Regist. Med: Technol. 36, 21-25.
In der Uzwiler-Studie (25) wurden Harnsäure-Bestimmungen an Proben von über 1400 Männern durchgeführt. Dabei wurde ein Mittelwert von $279 \mu \mathrm{mol} / 1$ mit einem $2 \mathrm{~s}-B e r e i c h$ von 134 bis $424 \mu \mathrm{mol} / \mathrm{l}$ getunden. Wenn man die Percentile mit Schrankenwerten von 10-90\% ermittelt, so ergeben sich für diese Kollektiv 196-372 $\mu \mathrm{mol} / 1$, mit einem Median von $274 \mu \mathrm{mol} / 1$.

In jüngster Zeit sind zwei größere Studien in ähnlicher Art in Berlin von Hoffmeister (28) und' in München von Zöllner (29) durchgeführt worden. In beiden Untersuchungen wurden enzymatische Verfahren zur Harnsäure-Bestimmung eingesetzt. Zöllner hat mit seiner Methode (4) für Männer den Bereich von 229-482 $\mu \mathrm{mol} / 1$ ermittelt, während in Berlin mit dem Kageyama-Verfahren (12) für Männer 202-416 $\mu \mathrm{mol} / 1$ errechnet wurden. Die eigenen Ergebnisse liegen demnach zwischen denen von Berlin und München.

Man darf daher mit Recht annehmen, daß HarnsäureBestimmungen mit Uricase-Verfahren, gleichgültig in welcher Modifikation, zu weitgehend identischen, präzisen und richtigen Resultate führen. Da dies bei den .,,chemischen "Oxidationsmethoden zur Harnsäurebestimmung nicht der Fall ist, sollten sie aus den klinischen Laboratorien eliminiert werden. Auch im Hinblick darauf, daß Uricase-Verfahren heute von vielen, auch von kleineren Laboratorien durchgeführt werden, scheint es nicht mehr erlaubt, die Phosphorwolframsäure-Methode im AutoAnalyzer des Kliniklabors zu verwenden.

Es gibt keinen Grund, dieses unspezifische Verfahren beizubehalten. Die - vergleichsweise geringen - höheren Unkosten, die bei Uricase-Verfahren anfallen, sind durch die höhere diagnostische Sicherheit gerechtfertigt.

17. Nishi, H. H. (1967), Clin. Chem. 13, 12-18.

18. Folin, O. \& Denis, W. (1912-13), J. Biol. Chem. 13, 469 bis 475 .

19. Johnstone, J. M. (1952), J. Clin. Pathol. 5, 317-318.

20. Henry, R. J., Sobel, C. \& Kim, J. (1957), Amer. J. Clin. Pathol. 28, 152-160.

21. Gochman, N. \& Schmitz, J. M. (1971), Clin. Chem. 17, 1154-1159.

22. Caraway, W. T. (1963), Stand. Meth. Clin. Chem. 4, 239 bis 247.

23. Keller, H. (1971), Diagnostik 4, 393-396.

24. Hjelm, M. (1968), Z. Anal. Chem. 243, 781-790.

25. Escher, M., Heyden, S., Christeller, S., Gasser, J. P., Keller; H., Ramsler, L. \& Geel, O., Schweiz. Med. Wochenschr., im Druck.

26. Rappoport, A. E. (Ed.) (1972), Quality Control in Clinical Chemistry, Huber, Bern.

27. Mertz, D. P. (1973), Klin. Wochenschr. 51, 96-98.

28. Thefeld, W., Hoffmeister, H., Busch, E. W., Koller, P. U. \& Vollmar, J. (1973), Deut. Med. Wochenschr. 98, 380-384.

29. Griebsch, A. \& Zöllner, N. (1973), diese Z. 11, 348-356.

30. Roseman, S. \& Dorfman, A. (1951), J. Biol. Chem. 192, 105-114. 